\title{
Polymyxin-B hemoperfusion in septic patients: analysis of a multicenter registry
}

Salvatore Lucio Cutuli ${ }^{* *}$, Antonio Artigas ${ }^{2}$, Roberto Fumagalli ${ }^{3}$, Gianpaola Monti ${ }^{3}$, Vito Marco Ranieri ${ }^{4}$, Claudio Ronco ${ }^{5}$, Massimo Antonelli ${ }^{1}$ and The EUPHAS 2 Collaborative Group

\begin{abstract}
Background: In 2010, the EUPHAS 2 collaborative group created a registry with the purpose of recording data from critically ill patients suffering from severe sepsis and septic shock treated with polymyxin-B hemoperfusion (PMX-HP) for endotoxin removal. The aim of the registry was to verify the application of PMX-HP in the daily clinical practice.

Methods: The EUPHAS 2 registry involved 57 centers between January 2010 and December 2014, collecting retrospective data of 357 patients (297 in Europe and 60 in Asia) suffering from severe sepsis and septic shock caused by proved or suspected infection related to Gram negative bacteria. All patients received atleast one cycle of extracorporeal endotoxin removal by PMX-HP.

Results: Septic shock was diagnosed in 305 (85.4\%) patients. The most common source of infection was abdominal (44.0\%) followed by pulmonary (17.6\%). Gram negative bacteria represented $60.6 \%$ of the pathogens responsible of infection. After $72 \mathrm{~h}$ from the first cycle of PMX-HP, some of the SOFA score components significantly improved with respect to baseline: cardiovascular (2.16 \pm 1.77 from $3.32 \pm 1.29, p<0.0001)$, respiratory $(1.95 \pm 0.95$ from $2.40 \pm 1.06, p<0.001)$ and renal $(1.84 \pm 1.77$ from $2.23 \pm 1.62, p=0.013)$. Overall 28 -day survival rate was $54.5 \%$ ( $60.4 \%$ in abdominal and $47.5 \%$ in pulmonary infection). Patients with abdominal infection treated with PMX-HP within $24 \mathrm{~h}$ from the diagnosis of septic shock had a 28-day survival rate of $64.5 \%$. Patients showing a significantly cardiovascular improvement after PMX-HP had a 28-survival rate of $75 \%$ in comparison to the $39 \%$ of patients who did not $(p<0.001)$. Cox regression analysis found the variation of cardiovascular, respiratory and coagulation SOFA to be independent covariates for 28-day survival. In European patients were observed a higher 28-day (58.8 vs. $34.5 \%$, $p=0.003)$, ICU ( 59 vs. $36.7 \%, p=0.006$ ) and hospital survival rate ( 53.2 vs. $35 \%, p=0.02$ ) than in Asian patients. However, the two populations were highly heterogeneous in terms of source of infection and severity scores at admission.

Conclusion: The EUPHAS 2 is the largest registry conducted outside Japan on the clinical use of PMX-HP in septic patients. Data analysis confirmed the feasibility of PMX-HP to treat septic patients in daily clinical practice, showing clinical benefits associated with endotoxin removal without significant adverse events related to the extracorporeal technique.
\end{abstract}

Keywords: Sepsis, Septic shock, Infection, EAA, Extracorporeal endotoxin removal, Polymyxin-B hemoperfusion

\section{Background}

Polymyxin-B hemoperfusion (PMX-HP) [1] is a blood purification technique designed to bind and neutralize circulating endotoxins by extracorporeal removal,

\footnotetext{
*Correspondence: sl.cutuli@gmail.com

${ }^{1}$ Department of Intensive Care and Anaesthesiology, Agostino Gemelli University Hospital, Catholic University of the Sacred Heart, Largo A. Gemelli, 8, 00168 Rome, Italy

Full list of author information is available at the end of the article
}

inhibiting the progression of the septic cascade mediated by lipopolysaccharide (LPS) [2-16], the core lipid portion of the Gram negative bacterial wall. Endotoxin is removed from the blood by extracorporeal hemoperfusion with a polymyxin-B-adsorbing cartridge that binds endotoxin through covalent and ionic bonds. This therapy was first introduced in Japan in 1994 and 10 years later it was introduced in Europe. 
The most common indication for PMX-HP has been septic shock due to abdominal infections. In case of impairment of the intestinal mucosa, LPS can translocate into the bloodstream. The consequent huge activation of the immune system can lead to the dysfunction of multiple organs until the loss of their function (MOF). The importance of endotoxin to determine the unfavorable evolution of sepsis related to Gram negative bacteria has been clearly demonstrated [2-15].

Several trials have been carried out to verify the hypothesis that removing circulating endotoxin from the blood of patients suffering from abdominal septic shock could improve their clinical outcome, in terms of organ dysfunction and mortality [4-15].

The Italian nationwide Early Use of Polymyxin B Hemoperfusion in Abdominal Septic Shock (EUPHAS) Randomized Control Trial (RCT) [15], demonstrated a significant improvement in cardiovascular response and outcome of patients treated by PMX-HP for severe sepsis and septic shock due to abdominal infections. On the other hand, the French ABDOMIX RCT [16] failed to demonstrate clinical benefits of PMX-HP application in a cohort of patients suffering from peritonitis-induced septic shock after emergency surgery.

Furthermore, PMX-HP has been applied in septic patients with source of infection different from the abdomen as respiratory system [17].

Due to the conflicting evidence given by EUPHAS and ABDOMIX RCTs in sepsis sustained by abdominal source of infection and new potential indication highlighted by case report on respiratory infections, the real application of PMX-HP in daily clinical practice is unclear.

In 2010, the EUPHAS 2 project [18] was launched, to create an international registry with the purpose of recording data from patients affected by Gram negative related severe sepsis and septic shock treated with PMXHP. The aim of the project was to evaluate the application of PMX-HP in the daily clinical practice, both in order to verify the reproducibility of data currently available in the literature and to evaluate the population actually elected for the therapy.

The EUPHAS 2 project is divided into two phases. The first retrospective phase aimed to collect data from at least 250 patients. The analysis of the data from this phase was used to optimize the data collection for the second prospective phase, which aims to collect data from a much larger patient population. The results from the second phase will be used to identify subpopulations of patients who may benefit from this treatment more than others.

A preliminary analysis of EUPHAS 2 retrospective phase [19] confirmed the results of EUPHAS RCT in abdominal septic patients. In the current paper are presented the full results from the first retrospective phase of EUPHAS 2.

\section{Methods}

The EUPHAS 2 project was a voluntary registry specifically conceived for the evaluation and analysis of PMXHP application in the real clinical life.

All the patients admitted to the 57 participating centers with suspected Gram negative related severe sepsis and septic shock unresponsive to standard care [20] were included in the registry. Forty-six centers were located in Europe (Italy, Spain and Switzerland), and eleven centers in Asia (India). Data collection started in January 2010 and ended in December 2014.

The definition of severe sepsis and septic shock followed the consensus guideline enounced by Surviving Sepsis Campaign [20].

The Gram negative etiology of sepsis was suspected in relation to the source of infection (the abdomen is colonized by Gram negative species) or proven by microbiological tests. In 24 out of 57 centers involved in the registry, LPS might be assessed on peripheral blood sample by the neutrophil-dependent chemiluminescence-based endotoxin activity assay (EAA) [24]. EAA quantifies endotoxin levels through a relative scale ranking from 0 to 1 . EAA is characterized by a sensitivity of $85.3 \%$, a specificity of $44 \%$, a negative predictive value of $98.6 \%$ for the exclusion of Gram negative infection and $94.8 \%$ for the exclusion of all infection [21]. Marshall et al. [21] demonstrated a progressive mortality increase related to endotoxin activity (EA) rising value. Highest mortality rate was observed for EA greater than 0.6, whereby it is considered the threshold to start the LPS removal.

PMX-HP is not ruled by specific guidelines and this therapy is recommended in severe sepsis and septic shock unresponsive to standard care [20], caused by proved or suspected infection related to Gram negative bacteria. All patients received fluid infusion and vasopressor therapy if needed [20] and independently to PMX-HP.

In all patients, PMX-HP was performed using Toraymyxin ${ }^{\circledR}$ cartridge (Toray Medical Company, Tokyo, Japan) connected to the patient through a 10-12 French veno-venous catheter inserted into the right or left femoral or internal jugular vein and the pump flow rate was 80-120 $\mathrm{mL} / \mathrm{min}$.

Each session of PMX-HP lasted $2 \mathrm{~h}$. In relation to clinical condition of the patient and EA (where available), the clinician decided to perform a second cycle of PMX-HP with a delay of $24 \mathrm{~h}$ from the first treatment.

Heparin intravenous administration as anticoagulant was set starting from a bolus of 3000 UI before the beginning of PMX-HP with a continuous infusion of $20 \mathrm{UI} /$ 
$\mathrm{kg} / \mathrm{h}$ during the treatment. The recommended dose of heparin could be modified in relation to the hemorrhagic risk of the patient.

Main data collection included: timing of diagnosis of severe sepsis/septic shock and treatment with PMX-HP; hemodynamic status; septic organ function impairment; outcome at 28 days, ICU and hospital discharge. Septic progressive organ function impairment was monitored with SOFA score [22-25], hemodynamic status was evaluated with mean arterial pressure (MAP), lactates concentration in the blood and vasopressor load expressed as the inotropic core [(dopamine dose $\mathrm{x} 1)+($ dobutamine dose $\mathrm{x} 1)+($ adrenaline dose $\mathrm{x} 100)+($ noradrenaline dose $x$ 100) + (phenylephrine dose $x$ 100), wherein all doses are expressed as $\mu \mathrm{g} / \mathrm{kg} / \mathrm{min}$ ]. SOFA score, inotropic score and lactates concentration in the blood were assessed at the time of the first PMX-HP $\left(t_{0}\right)$ and every $24 \mathrm{~h}$ until $72 \mathrm{~h}\left(t_{72}\right)$ from $t_{0}$.

Patients showing with a reduction of at least one point in cardiovascular SOFA between $t_{0}$ and $t_{72}$ were considered "cardiovascular responders"; otherwise, they were "cardiovascular non-responders".

Adverse events associated with PMX-HP were recorded and defined as tachycardia-heart rate (HR) $>100 \mathrm{bpm}$ or HR increase $>10 \%$ HR pre-treatment during $10^{\prime}$ from the beginning of PMX-HP, hypotension-MAP $<70 \mathrm{mmHg}$ or PAM reduction $>10 \%$ MAP pre-treatment during $10^{\prime}$ from the beginning of PMX-HP, bleeding-every type of hemorrhagic complication, cartridge clotting-coagulation of polymyxin-B fiber cartridge. Clotting of the PMX cartridge is an adverse event because it causes a premature interruption of the treatment (limiting its benefit) and blood lost for the patient.

IRBs and Ethical Committee of all centers approved data collection and the study.

\section{Statistical analysis}

Normality tests were performed using the Kolmogorov-Smirnov test. Quantitative normally distributed variables (APACHE II, SAPS II, SOFA score and body temperature) were presented as mean \pm standard deviation (SD) and non-normally distributed variables (age, time to treatment, vasopressors, EAA and biochemical data) as medians and the 25th-75th quartiles range. Univariate comparison was performed to compare variables between two groups. The organ dysfunction scores were compared using the unpaired $t$ test. Categorical variables were expressed as actual numbers and percentages. In all comparisons, $p<0.05$ was considered statistically significant.

Analysis was performed using $\mathrm{R}$ statistical software. Student's $t$ test, Wilcoxon test and Mann-Whitney test were used to compare means in case of normal paired or unpaired samples, non-normal paired samples and nonnormal unpaired samples, respectively. Fisher's exact test was carried out to evaluate significance in contingency tables. Multiple logistic regression was performed to evaluate the dependence of categorical variables on one or more predictors, and linear multiple regression was used to model the dependence of scalar variables on one

\section{Table 1 Demographic characteristics of patients treated with PMX-HP}

\begin{tabular}{|c|c|}
\hline Demographic characteristics & $N 357$ \\
\hline Age, years, mean $\pm S D$ & $63(51-72)$ \\
\hline Gender, male & $240(67.2)$ \\
\hline SAPS II at admission, mean \pm SD & $50.3 \pm 19.2$ \\
\hline APACHE $\|$ at admission, mean \pm SD & $21.8 \pm 7.2$ \\
\hline SOFA at admission, mean \pm SD & $10.9 \pm 3.5$ \\
\hline Incidence of shock & $305(85.4)$ \\
\hline \multicolumn{2}{|l|}{ Source of sepsis } \\
\hline Abdominal & $157(44)$ \\
\hline Pulmonary & $63(17.6)$ \\
\hline Urinary & $16(4.5)$ \\
\hline Cardiac & $23(6.4)$ \\
\hline Trauma & $19(5.3)$ \\
\hline Other $^{\mathrm{a}}$ & $79(22)$ \\
\hline Patients without microbiological cultures N (\%) & $139(38.9)$ \\
\hline Patients with microbiological cultures & $218(61.1)$ \\
\hline Negative microbiological cultures & $41(18.8)$ \\
\hline Positive microbiological cultures & $177(81.1)$ \\
\hline Gram positive only & $17(7.8)$ \\
\hline Gram negative only & $81(37.2)$ \\
\hline Fungi only & $13(6.0)$ \\
\hline Mixed including gram negatives & $51(23.4)$ \\
\hline Mixed not including gram negatives & $8(3.7)$ \\
\hline Patients with endotoxin activity assay, $N(\%)$ & $132(37)$ \\
\hline Abdominal & $47(35.6)$ \\
\hline Non-abdominal & $85(64.4)$ \\
\hline Without microbiology data & $33(25)$ \\
\hline Negative cultures & $12(9)$ \\
\hline Gram positive only & $7(5.3)$ \\
\hline Gram negative only & $43(32.6)$ \\
\hline Fungi only & $14(10.6)$ \\
\hline Mixed including gram negatives & $21(15.9)$ \\
\hline Mixed not including gram negatives & $3(2.2)$ \\
\hline Patients with 2 treatments, $N(\%)$ & $219(61)$ \\
\hline Patients with 1 treatment, $N(\%)$ & $138(39)$ \\
\hline 28 days survival & $180(54.5)$ \\
\hline ICU survival & $192(55.2)$ \\
\hline Hospital survival & $172(50)$ \\
\hline
\end{tabular}

Data are expressed as $N(\%)$ apart from otherwise indicated

a Other includes soft tissue infections, CVC-related infections, meningitides 
or more explanatory variables. Cox proportional hazard model was used to relate time-to-death to multiple covariates. For all of the statistics tests and models, a 0.05 significance level was considered.

\section{Results}

Demographic and clinical data of the 357 patients treated with PMX-HP are reported in Table 1. Data were recorded by 35 out of 57 centers with a median of patients per center of 9 [3-13] in Europe and 4.5 (2.7-11) in Asia.

Septic shock was diagnosed in 305 patients and severe sepsis in 52 patients. Eight patients had not data about vasopressor infused and they were considered presumably affected by severe sepsis. Main sources of infection were the abdomen (44 \%) and the lung (17.6\%).

EA was recorded in 18 out of 24 centers in which the assay was available. EAA was measured in 132 out of 357 patients $(37.0 \%)$ with a median value of $0.77(0.69$ $0.90)$ at $t_{0}$. EAA was $\geq 0.6$ in 120 patients and $<0.6$ in 12 patients, but the small number did not allowed any comparison between two groups.

Microbiological evidence of infection was documented in $174(49.5 \%)$ patients. Gram negative bacteria were isolated in $60.6 \%$ of the microbiological samples ( $37.2 \%$ mono-microbial and $23.4 \%$ poly-microbial). In 139 patients, microbiological results were either not or partly reported.

Each session of PMX-HP theoretically lasted $2 \mathrm{~h}$. No data about reason (except cartridge clotting) and timing of its premature interruption were recorded in the registry.

The majority of patients $(73.8 \%)$ were treated within $24 \mathrm{~h}$ from the onset of severe sepsis and septic shock; $13.2 \%$ of patients received the PMX-HP between 24 and $48 \mathrm{~h}$ after the diagnosis of severe sepsis and septic shock, while $12.9 \%$ of patients had a delay of more than 2 days.

\section{Safety of Polymyxin-B hemoperfusion}

Two cycles of PMX-HP were performed in 219 patients; 138 patients received only one treatment.

Twenty-six adverse events were recorded (two tachycardias, ten hypotensions, one bleeding, thirteen cartridge clottings) (Additional file 1: Table S12).

Among the PMX-HP clotted cartridge, in eight patients was not administered heparin bolus at the beginning of the treatment and a lower amount of continuous heparin infusion was prescribed as compared to the recommended standard dose. No information about heparin dosage was reported for the remaining five clotted cartridges.

None of these adverse events caused clinical worsening or death of the patients.
Table 2 Variables changes $72 \mathrm{~h}$ after PMX-HP

\begin{tabular}{llll}
\hline Patients & $\boldsymbol{t}_{\mathbf{0}} \boldsymbol{N}=\mathbf{3 5 7}$ & $\boldsymbol{t}_{\mathbf{7 2}} \boldsymbol{N}=\mathbf{2 9 9}$ & $\boldsymbol{p}$ (Wilcoxon) \\
\hline SOFA score & $12.4 \pm 4.2$ & $10.5 \pm 5.3$ & $<0.001$ \\
Cardiovascular SOFA & $3.32 \pm 1.29$ & $2.16 \pm 1.77$ & $<0.001$ \\
Renal SOFA & $2.23 \pm 1.62$ & $1.84 \pm 1.77$ & 0.013 \\
Hepatic SOFA & $1.22 \pm 1.28$ & $1.19 \pm 1.30$ & 0.80 \\
Respiratory SOFA & $2.40 \pm 1.06$ & $1.95 \pm 0.95$ & $<0.001$ \\
Coagulation SOFA & $1.33 \pm 1.29$ & $1.67 \pm 1.38$ & 0.004 \\
Inotropic score & $30(11.9-72.5)$ & $6.0(0.0-22)$ & $<0.001$ \\
Lactate, mmol/L & $3.4(1.9-6.0)$ & $1.9(1.3-2.9)$ & $<0.001$ \\
Platelets, $10^{3} / \mu \mathrm{L}$ & $117(56-220)$ & $86(40-163)$ & $<0.001$ \\
\hline
\end{tabular}

Normally distributed data are expressed as mean \pm SD and non-normally distributed data as median (interquartile range)

Italics indicates significant $p$ values

\section{Effect of PMX-HP on organ dysfunction}

SAPS II score was $48.0 \pm 16.6$ in patients suffering from severe sepsis versus $50.5 \pm 19.5$ in patients suffering from septic shock patients. SOFA score at $t_{0}$ was $8.1 \pm 2.9$ in patients suffering from severe sepsis versus $12.9 \pm 4.1$ in patients suffering from septic shock patients; $72 \mathrm{~h}$ from the beginning of PMX-HP, SOFA score decreased in patients suffering from septic shock, but not in patients suffering from severe sepsis $(10.7 \pm 5.4$ vs $8.9 \pm 4.3$, respectively).

Table 2 shows the variations of each SOFA component between $t_{0}$ and $t_{72}$.

A significant improvement of cardiovascular $\left(t_{0}=3.32 \pm 1.29\right.$ vs. $\left.t_{72}=2.16 \pm 1.77 ; p<0.001\right)$, respiratory $\left(t_{0}=2.40 \pm 1.06\right.$ vs. $\left.t_{72}=1.95 \pm 0.95 ; p<0.001\right)$ and renal $\left(t_{0}=2.23 \pm 1.62\right.$ vs. $\left.t_{72}=1.84 \pm 1.77 ; p=0.013\right)$ scores with a reduction of inotropic score $\left[t_{0}=30.0\right.$ $(11.9-72.5)$ vs. $\left.t_{72}=6.0(0.0-22.0) ; p<0.001\right]$ and lactate levels $\left[t_{0}=3.4(1.9-6.0)\right.$ vs. $\left.t_{72}=1.9(1.3-2.9) ; p<0.001\right]$ were observed after a follow-up of $72 \mathrm{~h}$. Only the coagulation score significantly increased $\left(t_{0}=1.33 \pm 1.29 \mathrm{vs}\right.$. $\left.t_{72}=1.67 \pm 1.38 ; p=0.004\right)$ together with platelet reduction $\left[t_{0}=117(56-220)\right.$ vs. $\left.t_{72}=86(40-163) ; p<0.001\right]$; both were not responsible of meaningful clinical worsening. These results were confirmed excluding patients dead at $72 \mathrm{~h}$ except for renal SOFA improvement (Additional file 1: Table S1).

\section{Length of stay and mortality}

Median ICU and hospital length of stay (LoS) were 16 days (8-29) and 28 days (16-49), respectively. Overall 28-day survival after PMX-HP was $54.5 \%$. Patients with abdominal sepsis treated within $24 \mathrm{~h}$ from the diagnosis of septic shock had a survival rate of $64.5 \%$.

A Cox regression model, stratified by age, was carried out to identify the independent covariates affecting 
Table 3 Cox regression on mortality stratified by age

\begin{tabular}{|c|c|c|c|c|c|c|}
\hline & \multicolumn{2}{|c|}{ Univariate } & \multirow[t]{2}{*}{$p$} & \multicolumn{2}{|c|}{ Multivariate } & \multirow[t]{2}{*}{$p$} \\
\hline & HR & $95 \% \mathrm{Cl}$ & & HR & $95 \% \mathrm{Cl}$ & \\
\hline \multicolumn{7}{|l|}{ Septic source } \\
\hline Abdominal versus respiratory & 1.56 & $0.93-2.63$ & 0.09 & 0.99 & $0.25-3.97$ & 0.99 \\
\hline Abdominal versus urinary & 0.47 & $0.14-1.61$ & 0.23 & 0.86 & $0.04-16.75$ & 0.92 \\
\hline Cardiovascular SOFA at $t_{0}$ & 1.17 & $0.98-1.41$ & 0.09 & 1.61 & $0.98-2.67$ & 0.06 \\
\hline Coagulation SOFA at $t_{0}$ & 1.10 & $0.95-1.28$ & 0.21 & 0.96 & $0.68-1.36$ & 0.83 \\
\hline Respiratory SOFA at $t_{0}$ & 1.14 & $0.94-1.39$ & 0.17 & 1.42 & $0.83-2.44$ & 0.20 \\
\hline Renal SOFA at $t_{0}$ & 0.97 & $0.81-1.17$ & 0.75 & 0.85 & $0.53-1.38$ & 0.52 \\
\hline Liver SOFA at $t_{0}$ & 1.17 & $0.98-1.39$ & 0.08 & 1.38 & $0.89-2.14$ & 0.15 \\
\hline$\triangle$ Cardiovascular SOFA & 1.39 & $1.20-1.62$ & $<0.001$ & 1.68 & $1.21-2.32$ & 0.002 \\
\hline$\triangle$ Coagulation SOFA & 1.46 & $1.13-1.90$ & 0.004 & 1.64 & $1.07-2.50$ & 0.022 \\
\hline$\triangle$ Respiratory SOFA & 1.26 & $1.01-1.56$ & 0.04 & 1.68 & $0.99-2.84$ & 0.05 \\
\hline$\triangle$ Renal SOFA & 1.29 & $0.94-1.76$ & 0.11 & 0.83 & $0.47-1.47$ & 0.51 \\
\hline$\triangle$ Liver SOFA & 1.74 & $1.20-2.52$ & 0.003 & 1.66 & $0.93-2.94$ & 0.08 \\
\hline Timing $^{\mathrm{a}}$ & 0.99 & $0.96-1.02$ & 0.61 & 0.95 & $0.88-1.01$ & 0.20 \\
\hline European versus asiatic & 0.53 & $0.33-0.86$ & 0.001 & 2.51 & $0.29-22.03$ & 0.41 \\
\hline
\end{tabular}

Italics indicates significant $p$ values

a Time between the diagnosis of severe sepsis/septic shock and PMX-HP

Table 4 Factors influencing the lack of cardiovascular response

\begin{tabular}{llcc}
\hline All patients & Odds ratio & $\mathbf{9 5} \% \mathbf{C l}$ & $\boldsymbol{p}$ \\
\hline Age & 0.99 & $0.97-1.02$ & 0.82 \\
Cardiovascular SOFA at $t_{0}$ & 1.51 & $1.07-2.13$ & $<0.019$ \\
Coagulative SOFA at $t_{0}$ & 0.96 & $0.75-1.24$ & 0.78 \\
Liver SOFA at $t_{0}$ & 1.09 & $0.83-1.42$ & 0.54 \\
Renal SOFA at $t_{0}$ & 1.17 & $0.88-1.55$ & 0.28 \\
Respiratory SOFA at $t_{0}$ & 0.54 & $0.39-0.75$ & $<0.001$ \\
European versus Asian & 1.38 & $0.43-4.42$ & 0.59 \\
\hline
\end{tabular}

Italics indicates significant $p$ values

28-day overall survival (Table 3). The variation of cardiovascular, respiratory and coagulation SOFA were identified as independent covariates for 28-day survival. No significant difference in survival was observed between patients receiving one or two treatments $(p=0.44)$.

Factors influencing the lack of cardiovascular response have been evaluated by a logistic regression (Table 4).

Cardiovascular and respiratory SOFA at $t_{0}$ were independent factors influencing cardiovascular response. Cardiovascular response was positively associated with the former [OR $1.51(1.07-2.13,95 \%$ CI with $p<0.019)$ ] and negatively with the latter [OR $0.54(0.39-0.75,95 \%$ CI with $p<0.001)$ ].

Cardiovascular SOFA at baseline between responders and non-responders $(3.7 \pm 0.7$ vs. $3.2 \pm 1.4, p \leq 0.001)$ was significantly different. Survival rates between cardiovascular responders and non-responders were 74.6 and $41.9 \%$, respectively $(p<0.001$, Additional file 2 : Figure S1). No significant differences in terms of age and SAPS II ( $48.6 \pm 19.2$ vs $51.2 \pm 19.2, p=0.32)$ were detected between the two subgroups (Additional file 1: Table S3).

The univariate Cox regression model identified the geographical origin as a factor influencing the mortality.

The characteristics of European and Asian patients are reported in Table 5.

Europeans were older [66 (54-74) years vs. 54 (41.3$62), p<0.001]$, their SOFA score at enrollment was lower (11.8 \pm 3.9 vs. $15.6 \pm 4.7, p<0.001)$, the length of ICU and hospital stay was shorter [ICU LoS 11 (1-123) days vs. 17 (1-213), $p<0.001$; hospital LoS 14.5 (2-140) days vs. 34 (2-407), $p<0.001$ ], and they had a higher survival rate at 28-day than Asian (Additional file 3: Figure S2), both in the ICU ( 59 vs. $36.7 \%, p=0.006)$ and in the hospital (53.2 vs. $35.0 \%, p=0.02)$.

The degree of cardiovascular response to PMX-HP treatment was similar between the two populations (47.4 \% in European compared to $50 \%$ in Asian patients), as well as the time between the diagnosis and start of the treatment.

\section{Discussion}

The EUPHAS 2 data collection represents the largest database on PMX-HP application describing its utilization in the real clinical life. EUPHAS 2 registry was created to describe the actual use of the PMX-HP in the 
Table 5 Characteristics of enrolled patients by geographical origin

\begin{tabular}{|c|c|c|c|}
\hline Characteristics of enrolled patients by origin & $\begin{array}{l}N=297 \\
\text { European }\end{array}$ & $\begin{array}{l}N=60 \\
\text { Asian }\end{array}$ & $p$ \\
\hline Age, years & $66(54-74)$ & $54(41.3-62)$ & $<0.001$ \\
\hline SOFA score at admission & $10.8 \pm 3.5$ & $12.3 \pm 3.4$ & 0.16 \\
\hline SAPS $\|$ at admission & $49.6 \pm 18.0$ & $53.8 \pm 25.2$ & 0.21 \\
\hline Incidence of shock N (\%) & $257(86.5)$ & $48(80)$ & 0.31 \\
\hline \multicolumn{4}{|l|}{ Septic source N (\%) } \\
\hline Abdominal & $142(47.8)$ & $15(25)$ & 0.002 \\
\hline Pulmonary & $42(14.1)$ & $21(35)$ & $<0.001$ \\
\hline Urinary & $13(4.4)$ & $3(5)$ & 0.90 \\
\hline Postoperative cardiac surgery & $22(7.4)$ & $1(1.7)$ & 0.17 \\
\hline Trauma & $19(6.4)$ & 0 & - \\
\hline Other & $59(19.9)$ & $20(33.3)$ & 0.03 \\
\hline Days from diagnosis to enrollment $N$ (range) & $1(0-2.0)$ & $1.0(0.0-1.0)$ & 0.45 \\
\hline SOFA score at enrollment & $11.8 \pm 3.9$ & $15.6 \pm 4.7$ & $<0.001$ \\
\hline Cardiovascular SOFA at $t_{0}$ & $3.4 \pm 1.2$ & $3.2 \pm 1.5$ & 0.38 \\
\hline Renal SOFA at $t_{0}$ & $2.2 \pm 1.6$ & $2.6 \pm 1.6$ & 0.08 \\
\hline Hepatic SOFA at $t_{0}$ & $1.1 \pm 1.2$ & $1.9 \pm 1.6$ & $<0.001$ \\
\hline Respiratory SOFA at $t_{0}$ & $2.3 \pm 1.0$ & $2.8 \pm 1.1$ & 0.002 \\
\hline Coagulation SOFA at $t_{0}$ & $1.3 \pm 1.3$ & $1.5 \pm 1.3$ & 0.17 \\
\hline Inotropic score at $t_{0}$ & $6.0(0.0-20.3)$ & $5.0(0.0-49.3)$ & 0.15 \\
\hline Lactate at $t_{0}, \mathrm{mmol} / \mathrm{L}$ & $1.9(1.2-2.8)$ & $2.7(1.5-5.2)$ & 0.18 \\
\hline Platelets at $t_{0}, 10^{3} / \mu \mathrm{L}$ & $84(35.3-166.8)$ & $90(55.0-129.0)$ & 0.12 \\
\hline SOFA score at $t_{72}$ & $10.2 \pm 5.1$ & $13.3 \pm 6.4$ & $<0.001$ \\
\hline Cardiovascular SOFA at $t_{72}$ & $2.2 \pm 1.8$ & $2.0 \pm 1.9$ & 0.64 \\
\hline Renal SOFA at $t_{72}$ & $1.8 \pm 1.8$ & $2.2 \pm 1.8$ & 0.92 \\
\hline Hepatic SOFA at $t_{72}$ & $1.1 \pm 1.3$ & $2.0 \pm 1.5$ & 0.006 \\
\hline Respiratory SOFA at $t_{72}$ & $1.9 \pm 0.9$ & $2.0 \pm 1.1$ & 0.92 \\
\hline Coagulation SOFA at $t_{72}$ & $1.7 \pm 1.4$ & $1.7 \pm 1.2$ & 0.72 \\
\hline Inotropic Score at $t_{72}$ & $6.0(0.0-20.3)$ & $5.0(0.0-49.3)$ & 0.76 \\
\hline Lactates at $t_{72}, \mathrm{mmol} / \mathrm{L}$ & $1.9(1.2-2.8)$ & $2.7(1.5-5.2)$ & 0.09 \\
\hline Platelets at $t_{72}, 10^{3} / \mu \mathrm{L}$ & $84(35.3-166.8)$ & $90(55.0-129.0)$ & 0.63 \\
\hline 28-day survival, N (\%) & $160(58.8)$ & $20(34.5)$ & 0.003 \\
\hline ICU survival, N (\%) & $170(59)$ & $22(36.7)$ & 0.006 \\
\hline Hospital survival, $N(\%)$ & $158(53.2)$ & $21(35)$ & 0.02 \\
\hline
\end{tabular}

Normally distributed data are expressed as mean \pm SD and non-normally distributed data as median (interquartile range)

Italics indicates significant $p$ values

real life collecting data from unselected populations of septic patiens. PMX-HP is a complementary retreatment applied to patients affected by severe sepsis and septic shock that not respond to standard care.

The registry lacked of data about fluid load administered and hemodynamic monitor directing fluid resuscitation. However, each center performed PMX-HP only after the application of international consensus recommendation for sepsis, as strategy of resuscitation.

Surprisingly, in 142 patients (42\%) with clinical diagnosis of severe sepsis or septic shock, microbiological culture results were not reported that, if not performed, indicates a poor compliance to the bundles recommendations [20].

In 68 patients, EA was high although microbiological analysis did not reveal the presence of Gram negative germs. We can only suspect that the measured EA came from a Gram negative component of infection, which was either not revealed in microbiological tests or it was caused by direct translocation of endotoxin due to sepsisinduced permeability of the gut.

PMX-HP seemed to be safe: no major adverse events were reported. Data analysis showed a significant platelet count reduction at $t_{72}$ without clinical worsening of the 
patients. The phenomenon observed could be a manifestation of the septic cascade evolution, which could alter platelet count and function, otherwise a complication of the extracorporeal technique. Data collected in the registry did not permit to establish a relationship between platelet decline and PMX-HP or other causes. The majority of patients who experienced a severe decline in platelet numbers were also receiving Renal Replacement Therapy.

Despite the retrospective nature of EUPHAS 2 data collection and the lack of a control group, the relevant number of patients allowed a reliable statistical analysis of PMX-HP feasibility in a real clinical context.

Recently published epidemiologic studies reported a 48.7 \% in-hospital mortality for septic shock in France [26], as well as a $44 \%$ hospital mortality for severe sepsis in Asian countries [27], having both the same period of time of EUPHAS 2. The comparison between EUPHAS 2 and epidemiologic studies in France [26] and Asia [27] showed a trend to clinical improvement without significant adverse event associated with endotoxin removal. Patients with abdominal infections treated within $24 \mathrm{~h}$ from the diagnosis of septic shock, had a 28-day survival of $64.5 \%$, which was comparable with $68 \%$ observed in EUPHAS [15].

Recently, Zhou et al. [28] extended the analysis of effectiveness to all types of extracorporeal therapies in sepsis RCTs and concluded that the effect on mortality was mainly driven by studies using PMX-HP.

Iwagami et al. [29] published a retrospective analysis on 590 patients treated with PMX-HP matched through a propensity score to 590 patients treated with standard care who had open abdominal surgery on the day of admission for perforation of lower gastrointestinal tract, requiring the infusion of vasopressors. The authors showed a 28 -day mortality of $17.1 \%$ (101 of 590) in the PMX-HP group and $16.3 \%$ (96 of 590) in the control group $(p=0.696)$. Data came from a nationwide inpatient database in Japan, showing no benefit of PMX-HP in a less severe population characterized by a low mortality rate. However, Payen et al. in the ABDOMIX randomized controlled trial [16] observed an absence of benefit from the use of PMX membranes to treat peritonitis-induced septic shock after emergency surgery.

In ABDOMIX, the 28-day mortality rate was close to recent clinical RCTs on selected patient population affected by septic shock [30] unlike EUPHAS 2, which is a registry without specific inclusion criteria. ABDOMIX excluded patients affected by life-threatening abdominal diseases included in EUPHAS 2 as ischemia, obstruction, inflammatory colitis and trauma. In ABDOMIX, only 81 over the 119 treated patients had completed two sessions of PMX-HP unlike EUPHAS
[15], in which all patients enrolled had completed the two PMX-HP planned with a definitely higher mortality in the control group. In ABDOMIX, the isolation of yeasts from peritoneum was more frequent in PMXtreated group than in controls: this kind of disease represents a severe life-threatening condition not susceptible to improve by the endotoxin-adsorption blood purification techniques.

EUPHAS 2 confirmed that PMX-HP is often used to treat non-abdominal infection. The respiratory source was in fact the second clinical condition most frequently observed in the study and in this context the efficacy of the PMX-HP seemed less efficient. ICU and hospital survival were quite similar between patients with abdominal and respiratory sepsis, but patients with respiratory infections showed a trend toward a higher mortality rate at 28 days (Additional file 1: Table S2).

Lower respiratory tract infections might have some differences compared to abdominal or urinary sepsis. Pulmonary sepsis seems to respond less to the two sessions of endotoxin removal because source control is substantially achieved through the antibiotic action and the resolution needs a longer time. Differently in patients with intra-abdominal sepsis, the source control is obtained through surgery and the concomitant PMX treatment, achieving a more rapid resolution.

Another limitation of EUPHAS 2 is the lack of information about patients comorbidities and early antibiotic therapy appropriately applied, which cannot be obtained from the phase 1 of the registry. The phase 2 will clarify the implication of these important factors related to the outcome.

The database included both 297 European and 60 Asian patients. Even if baseline characteristics of patients at admission were similar between the two groups except for the age (Asian were younger than European, respectively $51.6 \pm 14.7$ vs $62.1 \pm 14.8$ years, $p<0.001$ ), there was a significant difference at the enrollment to PMXHP in terms of SOFA score and septic source. European and Asiatic population were not comparable for the septic source identified: Abdominal sepsis was prevalent in European patients, and pulmonary sepsis was most frequent in Asian patients.

Asiatic patients had a SOFA score higher than European patients $(15.6 \pm 4.7$ vs $12.4 \pm 4.2$ respectively, $p<0.001)$, especially for respiratory and hepatic components. Phua et al. [27] reported a lower compliance with surviving sepsis campaign bundles in Asian ICUs, particularly during the first $24 \mathrm{~h}$. A lower adherence to bundles correlated with worsening clinical condition and higher mortality.

Both European and Asian patients showed a similar clinical effect of PMX-HP treatment on organ 
dysfunction, that is mainly a significant reduction of vasopressor dependence and an improvement in gas exchange.

Published papers have underlined the positive effect of endotoxin removal by PMX-HP on cardiovascular dysfunction both increasing the arterial pressure and reducing the need of vasopressors.

In EUPHAS 2 registry, the cardiovascular component of SOFA represented $26.7 \%$ of the overall score when the treatment was started. This means that enrolled patients were likely to have a high vasopressor load. Several studies showed that a higher vasopressor load is related to a higher risk of death in septic shock. A recent publication by Monti et al. [31] analyzed the response to PMXHP of patients affected by refractory septic shock. They concluded that an early hemodynamic improvement highlighted by a vasopressor load reduction, potentially attenuates the mortality rate. The Cox regression analysis of 28-day mortality recognized the variation between baseline and $72 \mathrm{~h}$ of cardiovascular and coagulation components of SOFA score as independent covariates.

The similar degree of cardiovascular response to the PMX-HP treatment did not reflect a similar effect on mortality in Europe and Asia (Additional file 4: Figure S3, Additional file 5: Figure S4). Asian patients showed a lower survival rate and a higher length of stay than European patients, both justified by the worse organ dysfunction at the beginning of PMX-HP despite a cardiovascular response similar to European patients. The worse outcome of Asian patients could be related to the lower adherence to sepsis guideline and the higher incidence of respiratory infection than European patients.

In conclusion, notwithstanding the retrospective nature of the present data collection and the lack of a control group, these data confirmed the feasibility of the PMX-HP application in the usual clinical context without life-threatening adverse events related to its application, as already described in previous RCTs and observational studies. The ongoing EUPHRATES RCT [32] will clarify the effective clinical collocation of PMX-HP in patients suffering from endotoxin-mediated septic shock.

\section{Additional files}

Additional file 1. Supplementary Material.

Additional file 2: Figure S1. Kaplan-Meier cardiovascular responders vs cardiovascular non responders.

Additional file 3: Figure S2. Kaplan-Meier European patients versus Asian patients.

Additional file 4: Figure S3. Kaplan-Meier plot for European "responders/non-responders".

Additional file 5: Figure S4. Kaplan-Meier plot for Asian "responders/ non-responders".

\section{Abbreviations}

EAA: endotoxin activity assay; EA: endotoxin activity; ICU: intensive care units; LPS: lipopolysaccharide; MOF: multiple organ failure; PMX-HP: polymyxin-B hemoperfusion; RCT: randomized controlled trial; SS: severe sepsis; SSh: septic shock; HR: heart rate; MAP: mean arterial pressure.

\section{Authors' contributions}

All authors contributed in each part of the paper, from the drafting of the protocol, to the collection and analysis of data, up to the drafting of the manuscript. All authors read and approved the final manuscript.

\section{Author details \\ ${ }^{1}$ Department of Intensive Care and Anaesthesiology, Agostino Gemelli University Hospital, Catholic University of the Sacred Heart, Largo A. Gemelli, 8, 00168 Rome, Italy. ${ }^{2}$ Critical Care Center, Sabadell Hospital, CIBER Enferme- dades Respiratorias, Corporació Sanitària Universitària Parc Taulí, Universitat Autònoma de Barcelona, Sabadell, Spain. ${ }^{3}$ Department of Anaesthesia and Intensive Care Medicine, Niguarda Ca' Granda Hospital, University of Milan-Bicocca, Milan, Italy. ${ }^{4}$ Department of Anaesthesia and Intensive Care Medicine, University of Rome "La Sapienza", Rome, Italy. ${ }^{5}$ Department of Neph- rology, Dialysis and Transplantation, International Renal Research Institute of Vicenza (IRRIV), San Bortolo Hospital, Vicenza, Italy.}

\section{Acknowledgements}

The Early Use of Polymyxin B Hemoperfusion in the Abdominal Sepsis 2 Collaborative Group: Riccardo Maviglia, Sandra Cicconi, Davide Silvestri, Giuseppe Bello, Alessandra Brendolan, Federico Nalesso, Gianluca Villa, Pasquale Piccinni, Erica Martin, Vincenzo Cantaluppi, Sergio Vesconi, Giampaolo Casella, Egidio Fasanella, Michele Debitonto, Gianmario Monza, Angelo Blasetti, Rosaria Coletta, Michele D'Ambrosio, Gilda Cinnella, Patrizia Murino, Eugenio Piscitelli, Gaetano Centonze, Marco Cucurachi, Giuseppe Altieri, Vincenzo Leonardo, Anna Sara Idra, Goffredo Del Rosso, Maria Polidoro, Nicola Stigliano, Giuseppe Pittella, Gianluca Paternoster, Giuseppe Pulito, Daniela Puscio, Diego Cingolani, Gabriele Falzetti, Pietro Vecchiarelli, Francesco Giunta, Francesco Forfori, Giacomo Castiglione, Stefano Greco, Carlo Capra, Luciano Crema, Leonor Tamayo, Cristina Urbano, Brunello Pezza, Nadia Zarrillo, Pasquale Di Monaco, Giuseppe Climaco, Pasquale De Negri, Pasqualina Modano, Riccardo Pagliarulo, Claudio Petrillo, Tania Stripoli, Roberto Oggioni, Laura Campiglia, Anna Rita Valletta, Manuela Lugano, Domenico Milella, Laura Micucci, Ursula Reist, Rolf Ensner, Christian Gianbarba, Lukas Brander, Rajib Paul, Rajesh Crawla, Sanjeev Jasujia, Rajesh Pande, Pratibha Dileep, Sankaran Sundar, Raju Ganesan, Sandeep Dewan, Vivek Nangia, Raj Kumar Mani, Omender Singh, Pracee Sathe, Gupta Sachin, Pradeep M. D'Costa, Samavedam Srivanas, Yogendra Pal Singh, Kent Doi, Fumika Taki, Ricard Ferrer Roca, Eduardo Romay Medina, Josè Gernacho, Francisco Martí, Alberto Martinez-Ruiz, Fernando Martinez-Sagasti, Rafael Zaragoza Crespo, Paola Torti, Valeria Terzi.

\section{Competing interests}

The authors MA, SLC, VMR received an unrestricted research grant from ESTOR s.p.a. that funded part of the EUPHAS study published in the JAMA; AM is a member of the steering committee of the Euphrates study; AA received an unrestricted research grant from Ferrer Pharma, Grifols, Rubio, AM Pharma; CR received an unrestricted research grant from Toray, GE, Asahi, Astute.

\section{Ethics approval and consent to participate}

IRBs and Ethical Committee of all centers approved data collection and the study. Informed consent was waived due to the retrospective nature of the data.

\section{Funding}

The study was partially supported by ESTOR s.p.a.

Received: 23 March 2016 Accepted: 18 July 2016

Published online: 08 August 2016

\section{References}

1. Shoji H. Extracorporeal endotoxin removal for the treatment of sepsis: endotoxin adsorption cartridge (Toraymyxin). Ther Apher Dial. 2003;7:108-14 
2. Opal SM, Gluck T. Endotoxin as a drug target. Crit Care Med. 2003;31:S57-64.

3. Kellum JA. A targeted extracorporeal therapy for endotoxemia: the time has come. Crit Care. 2007:11:137.

4. Marshall JC, Foster D, Vincent JL, Cook DJ, Cohen J, Dellinger RP, Opal S, Abraham E, Brett SJ, Smith T, Mehta S, Derzko A, Romaschin A. Diagnostic and prognostic implications of endotoxemia in critical illness: results of the MEDIC study. J Infect Dis. 2004;190:527-34.

5. Perego AF, Morabito S, Graziani G, Casella GP, Parodi O. Polymyxin-B direct hemoperfusion (PMX-DHP) in gram negative sepsis. G Ital Nefrol. 2006:23(Suppl 36):S94-102.

6. Nakamura T, Ebihara I, Shoji H, Ushiyama C, Suzuki S, Koide H. Treatment with polymyxin B-immobilized fiber reduces platelet activation in septic shock patients: decrease in plasma levels of soluble P-selectin, platelet factor 4 and beta-thromboglobulin. Inflamm Res. 1999:48:171-5.

7. Cantaluppi V, Assenzio B, Pasero D, Romanazzi GM, Pacitti A, Lanfranco G, Puntorieri V, Martin EL, Mascia L, Monti G, Casella G, Segoloni GP, Camussi G, Ranieri VM. Polymyxin-B hemoperfusion inactivates circulating proapoptotic factors. Intensive Care Med. 2008;34:1638-45.

8. Kushi H, Miki T, Okamaoto K, Nakahara J, Saito T, Tanjoh K. Early hemoperfusion with an immobilized polymyxin B fiber column eliminates humoral mediators and improves pulmonary oxygenation. Crit Care. 2005;9:R653-61.

9. Suzuki H, Nemoto H, Nakamoto H, Okada H, Sugahara S, Kanno Y, Moriwaki K. Continuous hemodiafiltration with Polymyxin-B immobilized fiber is effective in patients with sepsis syndrome and acute renal failure. Ther Apher. 2002;6:234-40.

10. Uriu K, Osajima A, Hiroshige K, Watanabe H, Aibara K, Inada Y, Segawa K, Anai H, Takagi I, Ito A, Kamochi M, Kaizu K. Endotoxin removal by direct hemoperfusion with an adsorbent column using polymyxin B-immobilized fiber ameliorates systemic circulatory disturbance in patients with septic shock. Am J Kidney Dis. 2002;39:937-47.

11. Nemoto H, Nakamoto H, Okada H, Sugahara S, Moriwaki K, Arai M, Kanno Y, Suzuki H. Newly developed immobilized polymyxin B fibers improve the survival of patients with sepsis. Blood Purif. 2001;19:361-8.

12. Nakamura H, Matsuda K, Suzuki Y, Shoji H, Koide H. Polymyxin B-immobilized fiber hemoperfusion in patients with sepsis. Dial Transplant. 2003;32:602-7.

13. Ruberto F, Pugliese F, D'Alio A, Martelli S, Bruno K, Marcellino V, Summonti D, Celli P, Perrella S, Cappannoli A, Pietropaoli C, Tosi A, Diana B, Novelli G, Rossi M, Ginanni-Corradini S, Ferretti G, Berloco PB, Pietropaoli P. Clinical effects of direct hemoperfusion using a polymyxin-B immobilized column in solid organ transplanted patients with signs of severe sepsis and septic shock. A pilot study. Int J Artif Org. 2007;30:915-22.

14. Cruz DN, Perazella MA, Bellomo R, de Cal M, Polanco N, Corradi V, Lentini P, Nalesso F, Ueno T, Ranieri VM, Ronco C. Effectiveness of polymyxin B-immobilized fiber column in sepsis: a systematic review. Crit Care. 2007:11:R47.

15. Cruz DN, Antonelli M, Fumagalli R, Foltran F, Brienza N, Donati A, Malcangi V, Petrini F, Volta G, Bobbio Pallavicini FM, Rottoli F, Giunta F, Ronco C. Early use of polymyxin B hemoperfusion in abdominal septic shock: the EUPHAS randomized controlled trial. JAMA. 2009;301:2445-52.

16. Payen DM, Guilhot J, Launey Y, Lukaszewicz AC, Kaaki M, Veber B, Pottecher J, Joannes-Boyau O, Martin-Lefevre L, Jabaudon M, Mimoz O, Coudroy R, Ferrandiere M, Kipnis E, Vela C, Chevallier S, Mallat J, Robert R. Early use of polymyxin B hemoperfusion in patients with septic shock due to peritonitis: a multicenter randomized control trial. Intensive Care Med. 2015;41 (6):975-84.

17. Tsushima K, Kubo K, Yoshikawa S, Koizumi T, Yasuo M, Furuya S, Hora K. Effects of PMX-DHP treatment for patients with directly induced acute respiratory distress syndrome. Ther Apher Dial. 2007;11(2):138-45.

18. Martin EL, Cruz DN, Monti G, Casella G, Vesconi S, Ranieri VM, Ronco C, Antonelli M. Endotoxin removal: how far from the evidence? The EUPHAS 2 Project. Contrib Nephrol. 2010;167:119-25.
19. Early Use of Polymyxin B Hemoperfusion in the Abdominal Sepsis 2 Collaborative Group. Polymyxin B hemoperfusion in clinical practice: the picture from an unbound collaborative registry. Blood Purif. 2014;37(Suppl 1):22-5.

20. Dellinger RP, Levy MM, Rhodes A, Annane D, Gerlach H, Opal SM, Sevransky JE, Sprung CL, Douglas IS, Jaeschke R, Osborn TM, Nunnally ME, Townsend SR, Reinhart K, Kleinpell RM, Angus DC, Deutschman CS, Machado FR, Rubenfeld GD, Webb SA, Beale RJ, Vincent JL, Moreno R. Surviving sepsis campaign: international guidelines for management of severe sepsis and septic shock: 2012. Crit Care Med. 2013;41:580-637.

21. Marshall JC, Walker PM, Foster DM, Harris D, Ribeiro M, Paice J, Romaschin $A D$, Derzko AN. Measurement of endotoxin activity in critically ill patients using whole blood neutrophil dependent chemiluminescence. Crit Care. 2002;6:342-8

22. Vincent JL, Moreno R, Takala J, Willatts S, De Mendonca A, Bruining H, Reinhart CK, Suter PM, Thijs LG. The SOFA (Sepsis-related Organ Failure Assessment) score to describe organ dysfunction/failure. On behalf of the Working Group on Sepsis-Related Problems of the European Society of Intensive Care Medicine. Intensive Care Med. 1996;22:707-10.

23. Ferreira FL, Bota DP, Bross A, Melot C, Vincent JL. Serial evaluation of the SOFA score to predict outcome in critically ill patients. JAMA. 2001:286:1754-8.

24. Moreno R, Vincent IL, Matos R, Mendonca A, Cantraine F, Thijs L, Takala J, Sprung C, Antonelli M, Bruining H, Willatts S. The use of maximum SOFA score to quantify organ dysfunction/failure in intensive care. Results of a prospective, multicentre study. Working Group on Sepsis related Problems of the ESICM. Intensive Care Med. 1999;25:686-96.

25. Jones AE, Trzeciak S, Kline JA. The Sequential Organ Failure Assessment score for predicting outcome in patients with severe sepsis and evidence of hypoperfusion at the time of emergency department presentation. Crit Care Med. 2009:37:1649-54.

26. Quenot JP, Binquet C, Kara F, Martinet O, Ganster F, Navellou JC, Castelain V, Barraud D, Cousson J, Louis G, Perez P, Kuteifan K, Noirot A, Badie J, Mezher C, Lessire H, Pavon A. The epidemiology of septic shock in French intensive care units: the prospective multicenter cohort EPISS study. Crit Care. 2013;17:R65.

27. Phua J, Koh Y, Du B, Tang YQ, Divatia JV, Tan CC, Gomersall CD, Faruq MO, Shrestha BR, Gia Binh N, Arabi YM, Salahuddin N, Wahyuprajitno B, Tu ML, Wahab AY, Hameed AA, Nishimura M, Procyshyn M, Chan YH. Management of severe sepsis in patients admitted to Asian intensive care units: prospective cohort study. BMJ. 2011;342:d3245.

28. Zhou F, Peng Z, Murugan R, Kellum JA. Blood purification and mortality in sepsis: a meta-analysis of randomized trials. Crit Care Med. 2013:41:2209-20.

29. Iwagami M, Yasunaga H, Doi K, Horiguchi H, Fushimi K, Matsubara T, Yahagi N, Noiri E. Postoperative polymyxin B hemoperfusion and mortality in patients with abdominal septic shock: a propensity-matched analysis. Crit Care Med. 2014;42(5):1187-93.

30. Angus DC, Barnato AE, Bell D, Bellomo R, Chong CR, Coats TJ, Davies A, Delaney A, Harrison DA, Holdgate A, Howe B, Huang DT, Iwashyna T, Kellum JA, Peake SL, Pike F, Reade MC, Rowan KM, Singer M, Webb SA, Weissfeld LA, Yealy DM, Young JD. A systematic review and meta-analysis of early goal-directed therapy for septic shock: the ARISE, ProCESS and ProMISe Investigators. Intensive Care Med. 2015;41(9):1549-60.

31. Monti G, Terzi V, Calini A, Di Marco F, Cruz D, Pulici M, Brioschi P, Vesconi S, Fumagalli R, Casella G. Rescue therapy with polymyxin B hemoperfusion in high-dose vasopressor therapy refractory septic shock. Miner Anestesiol. 2015;81:516-25.

32. Klein DJ, Foster D, Schorr CA, Kazempour K, Walker PM, Dellinger RP. The EUPHRATES trial (Evaluating the Use of Polymyxin B Hemoperfusion in a Randomized controlled trial of Adults Treated for Endotoxemia and Septic shock): study protocol for a randomized controlled trial. Trials. 2014;11(15):218. doi:10.1186/1745-6215-15-218. 